\title{
EFFECTIVE SOLUTIONS OF SOME DUAL INTEGRAL EQUATIONS AND THEIR APPLICATIONS
}

\author{
ELENA OBOLASHVILI \\ Institute of Mathematics, Georgian Academy of Sciences \\ Tbilisi, Georgia \\ E-mail: helen@imath.kheta.ge
}

\begin{abstract}
Integral equations of the form (2) below, dual to (1) are studied from the point of view of finding their effective solutions, the results being given in Section 1. The results are applied in Section 2 for solving nonlocal problems for the polyharmonic functions in the half plane.
\end{abstract}

Introduction. In $\left[5_{a}\right]$ solutions of certain nonlocal problems for elliptic differential equations are reduced to the classical Wiener-Hopf integral equation and dual integral equations of convolution type. Effective solutions of such equations are well known [3], [7]. These equations are generalized by many investigators. A highly detailed survey of them can be found in [3], where some generalized integral equations are solved effectively and some of them are investigated in the sense of solvability. In $\left[5_{b}\right]$ the nonlocal problems for elliptic differential equations are reduced to integral equations of the form

$$
\varphi(x)+\int_{0}^{\infty}[k(x-t)+m k(x+t)] \varphi(t) d t=f(x), \quad x>0,
$$

and its solutions are constructed in quadratures when $m^{2}=1$, of course for $m=0$ too, as the classical Wiener-Hopf equation. The general form of equation (1) is considered in $[2],[6]$.

Dual integral equations

$$
\begin{aligned}
& \varphi(x)+\int_{\mathbb{R}}\left[k_{1}(x-t)+p_{1}(x+t)\right] \varphi(t) d t=f_{1}(x), \quad x>0, \\
& \varphi(x)+\int_{\mathbb{R}}\left[k_{2}(x-t)+p_{2}(x+t)\right] \varphi(t) d t=f_{2}(x), \quad x<0,
\end{aligned}
$$

1991 Mathematics Subject Classification: Primary 46C20; Secondary 32G81.

The paper is in final form and no version of it will be published elsewhere. 
are written in [1], but cases of their effective solutions are not indicated there. The case $k_{1}=k_{2}, p_{1}=p_{2}$ is the simplest and using Fourier integral transform their solution can be written immediately [7]. It is interesting to note that in our applications just such equations are obtained when the solutions are represented explicitly.

1. Dual integral equations. Let $L_{2} H$ be the class of square integrable and Holdercontinuous functions on the real axis $\mathbb{R}$. The class of functions, whose Fourier integral transforms on $\mathbb{R}$ belong to the class $L_{2} H$, will be denoted by $F L_{2} H$.Some nonlocal problems for poliharmonic functions in the halfplane considered below are reduced to the dual integral equations of the form

$$
\begin{aligned}
& \varphi(x)+\frac{1}{\sqrt{2 \pi}} \int_{\mathbb{R}} \varphi(t)\left[k_{1}(x-t)+m k_{1}(x+t)\right] d t=f_{1}(x), \quad x>0, \\
& \varphi(x)+\frac{1}{\sqrt{2 \pi}} \int_{\mathbb{R}} \varphi(t)\left[k_{2}(x-t)+m k_{2}(x+t)\right] d t=f_{2}(x), \quad x<0,
\end{aligned}
$$

where $m \neq 0$ is a real constant, $\varphi(x)$ is an unknown function and $k_{1}, k_{2}, f_{1}, f_{2}$ are given functions belonging to $F L_{2} H$. When $m=0$ the solution of (3) is represented effectively in [3] using the Fourier integral transform and the Riemann boundary value problem for one piecewise holomorphic function of one complex variable. As above was noted, in the case $k_{1}=k_{2}$ equations (3) are the simplest ones. Thus we assume later on that

$$
m \neq 0, \quad k_{1} \neq k_{2} \text {. }
$$

We shall see in the sequel that for $m^{2}=1$ the equations (3) can be solved effectevely. This case is just needed for our applications. Solution of $(3)$. Let $\widehat{f}(x)$ be the Fourier integral transform of $f(x) \in F L_{2} H$ :

$$
\widehat{f}(x) \equiv F_{x}[f(t)]=\frac{1}{\sqrt{2 \pi}} \int_{\mathbb{R}} f(t) e^{i x t} d t, \quad x \in \mathbb{R} .
$$

As is well known, for the convolution $f \star k$ is true the equalities:

$$
\begin{gathered}
F_{x}[f \star k]=\widehat{f}(x) \widehat{k}(x) \\
F_{x}\left[\frac{1}{\sqrt{2 \pi}} \int_{\mathbb{R}} k(x+t) f(t) d t\right]=\widehat{k}(x) \widehat{f}(-x)
\end{gathered}
$$

equations (3) rewrite as

$$
\begin{gathered}
\varphi(x)+\frac{1}{\sqrt{2 \pi}} \int_{\mathbb{R}} \varphi(t)\left[k_{1}(x-t)+m k_{1}(x+t)\right] d t=f_{1}(x)+f_{-}(x) \\
\varphi(x)+\frac{1}{\sqrt{2 \pi}} \int_{\mathbb{R}} \varphi(t)\left[k_{2}(x-t)+m k_{2}(x+t)\right] d t=f_{2}(x)+f_{+}(x), \quad x \in \mathbb{R},
\end{gathered}
$$

where $f_{+}(x), f_{-}(x)$ are the additional unknown rightsided and leftsided functions, respectively: $f_{+}(x)=0$ for $x<0$ and $f_{+}(x) \neq 0$ for $x>0 ; f_{-}(x)$ is defined vice versa. It is easy to see (for example, [3]) that

$$
F_{x}\left[f_{+}(t)\right]=F^{+}(x), \quad F_{x}\left[f_{-}(t)\right]=F^{-}(x), \quad x \in \mathbb{R}
$$


are the boundary values of some function $F(z), \quad(z=x+i y)$, holomorphic in the upper halfplane $y>0$ and in the lower halfplane $y<0$, respectively.

Applying the Fourier integral transform to (7) and take into consideration (6) and (8), we shall receive the system of equations

$$
\begin{aligned}
{\left[1+\widehat{k}_{1}(x)\right] \widehat{\varphi}(x)+m \widehat{k}_{1}(x) \widehat{\varphi}(-x) } & =\widehat{f}_{1}(x)+F^{-}(x) \\
{\left[1+\widehat{k}_{2}(x)\right] \widehat{\varphi}(x)+m \widehat{k}_{2}(x) \widehat{\varphi}(-x) } & =\widehat{f}_{2}(x)+F^{+}(x) . x \in \mathbb{R}
\end{aligned}
$$

By force of the conditions (4)

$$
D(x)=m\left[\widehat{k}_{2}(x)-\widehat{k}_{1}(x)\right] \neq 0 .
$$

Thus, by (9) one can define uniquely $\widehat{\varphi}(x)$ and $\widehat{\varphi}(-x)$.

Replace $-x$ by $x$ in the expression of $\widehat{\varphi}(-x)$ and equate it to $\widehat{\varphi}(x)$ defined from (9), then we shall obtain the boundary condition for piecewise holomorphic function $F(z)$ :

$$
\begin{gathered}
\frac{m}{D(x)}\left[\widehat{k}_{2}(x) F^{-}(x)-\widehat{k}_{1}(x) F^{+}(x)\right]= \\
=\frac{1}{D(-x)}\left[\left(1+\widehat{k}_{1}(-x)\right) F^{+}(-x)-\left(1+\widehat{k}_{2}(-x)\right) F^{-}(-x)\right]+g_{1}(x), \quad x \in \mathbb{R},
\end{gathered}
$$

where $g_{1}(x)$ is the known function defined by $\widehat{f}_{1}$ and $\widehat{f}_{2}$.

Consider two piecewise holomorphic functions:

$$
F_{1}(z)= \begin{cases}F(z), & y>0 \\ F(-z), & y<0\end{cases}
$$

and

$$
F_{2}(z)= \begin{cases}F(-z), & y>0 \\ F(z), & y<0\end{cases}
$$

Thus

$$
F_{1}^{+}(x)=F^{+}(x), \quad F_{1}^{-}(x)=F^{+}(-x), \quad F_{2}^{+}(x)=F^{-}(-x), \quad F_{2}^{-}(x)=F^{-}(x) .
$$

Then (11) and the equality, obtained from (11), where $x$ is replaced by $-x$, can be written in the form

$$
\begin{gathered}
\frac{m \widehat{k}_{1}(x)}{D(x)} F_{1}^{+}(x)-\frac{1+\widehat{k}_{2}(-x)}{D(-x)} F_{2}^{+}(x)= \\
-\frac{1+\widehat{k}_{1}(-x)}{D(-x)} F_{1}^{-}(x)++\frac{m \widehat{k}_{2}(x)}{D(x)} F_{2}^{-}(x)-g_{1}(x) \\
\frac{1+\widehat{k}_{1}(x)}{D(x)} F_{1}^{+}(x)-\frac{m \widehat{k}_{2}(-x)}{D(-x)} F_{2}^{-}(x)=-\frac{m \widehat{k}_{1}(-x)}{D(-x)} F_{1}^{-}(x)+ \\
+\frac{1+\widehat{k}_{2}(x)}{D(x)} F_{2}^{-}(x)-g_{1}(-x), \quad x \in \mathbb{R} .
\end{gathered}
$$

Thus, we have the Riemann problem for two piecewise holomorphic functions $F_{1}(z)$ and $F_{2}(z)$. This system can not in general be solved effectively. But fortunately, when $m^{2}=1$ 
it can be done so. We shall consider just this case. Let

$$
D_{1}(x) \equiv \frac{1+\widehat{k}_{1}(x)+\widehat{k}_{2}(-x)}{D(x) D(-x)} \neq 0, \quad x \in \mathbb{R} .
$$

Then the system (14) can be rewritten in an equivalent form

$$
\begin{gathered}
F_{1}^{+}(x)=\frac{1}{D_{1}(x)}\left[\frac{1}{D(-x)} F_{1}^{-}(x)+\frac{1+\widehat{k}_{2}(x)+\widehat{k}_{2}(-x)}{D(x) D(-x)} F_{2}^{-}(x)\right]+d_{1}(x), \\
F_{2}^{+}(x)=\frac{1}{D_{1}(x)}\left[\frac{1+\widehat{k}_{1}(-x)+\widehat{k}_{1}(x)}{D(x) D(-x)} F_{1}^{-}(x)-\frac{1}{D(x)} F_{2}^{-}(x)\right]+d_{2}(x), \quad x \in \mathbb{R} .
\end{gathered}
$$

where $d_{1}, d_{2}$ are given functions.

It is easy to obtain the following Riemann boundary value problem for one piecewise holomorphic function, taking into consideration (10) and (16): In the case $m=1$

$$
F_{1}^{+}(x)+F_{2}^{+}(x)=G(x)\left[F_{1}^{-}(x)+F_{2}^{-}(x)\right]+g(x), \quad x \in \mathbb{R},
$$

and in the case $m=-1$

$$
F_{1}^{+}(x)-F_{2}^{+}(x)=G(x)\left[F_{1}^{-}(x)-F_{2}^{-}(x)\right]+g(x), \quad x \in \mathbb{R},
$$

where

$$
G(x)=\frac{1+\widehat{k}_{2}(x)+\widehat{k}_{1}(-x)}{1+\widehat{k}_{2}(-x)+\widehat{k}_{1}(x)}
$$

Thus $G(\infty)=G(-\infty)=1$ and by force of $(15) G(x) \neq 0, x \in \mathbb{R}$

Hence, as is well known [4], by these conditions piecewise holomorphic functions $F_{1}(z)+F_{2}(z)$ and $F_{1}(z)-F_{2}(z)$ will be defined in quadratures. After this, defining $F_{2}(z)$ with the help of $F_{1}(z)$ and putting it in the first condition of (16), we shall obtain again the Riemann boundary value problem for one piecewise function $F_{1}(z)$. Therefore both functions $F_{1}(z)$ and $F_{2}(z)$ will be defined perfectly. Next, $F(z)$ will be defined by (12)(13) and $\widehat{\varphi}(x)$ by equations (9). Hence, the solution of the dual integral equations (3) $\varphi(x)$ will be defined by $\widehat{\varphi}(x)$ using the inverse Fourier transform formula.

2. Nonlocal problems for polyharmonic functions in the halfplane. Let $D$ be a halfplane $(y>0)$ and $u(x, y)$ be a regular solution of the equation

$$
\Delta^{n} u=0, \quad(n \geq 1) .
$$

Problem 1 . Define in $D$ the function $u(x, y)$ vanishing at infinity by the $(n-1)$ conditions on $\mathbb{R}$

$$
\left.v_{k}(x, 0) \equiv \frac{\partial^{k} u}{\partial y^{k}}\right|_{y=0}=f_{k}(x), \quad k=0,1, \ldots, l-1, l+1, \ldots n-1,
$$

and, besides, the following nonlocal conditions:

$$
\begin{gathered}
v_{l}(x, 0)=v_{l}\left(x, h_{1}\right)+m v_{l}\left(-x, h_{1}\right)+f_{l}(x), \quad x>0 \\
v_{l}(x, 0)=v_{l}\left(x, h_{2}\right)+m v_{l}\left(-x, h_{2}\right)+f_{l}(x), \quad x<0,
\end{gathered}
$$


where $h_{1} \neq h_{2}$ are positive constants, $m^{2}=1, l$ is fixed $0 \leq l \leq n-1$, all $f_{k}(x) \in F L_{2} H$. The case $h_{1}=h_{2}$ is very simple and the solution can be written easily.

Problem 2. Define in $D$ the function $u(x, y)$ vanishing at infinity by the $n-1$ boundary conditions on $\mathbb{R}$

$$
\left.v_{k}(x, 0) \equiv \Delta^{k} u\right|_{y=0}=f_{k}(x), \quad x \in \mathbb{R}, \quad k=0,1, \ldots, l-1, l+1, \ldots, n-1,
$$

and for $v_{l}(x, 0)$ have the nonlocal conditions of the form (22).

Problem 3 . Define in $D$ the function $u(x, y)$ vanishing at infinity by the boundary conditions (21) on $\mathbb{R}$ and, besides, the nonlocal conditions:

$$
\begin{gathered}
v_{l}(x, 0)=f_{l}(x), \quad x<0 \\
v_{l}(x, 0)=v_{l}(x, h)+m v_{l}(-x, h)+f_{l}(x), \quad x>0,
\end{gathered}
$$

where $h>0$ is given constant, $f_{l}(x)$ is given function too.

Note that the case $m=0$ were considered in $\left[5_{a}\right]$ and the problem is solved explicitely.

Solution of the problem 1 . First of all we need the solution of the classical problem for (20) in $D$ when on $\mathbb{R}$ is given (21) for all $k=0,1, \ldots, n-1$. It can be written in the form (see, for example, $\left[5_{a}\right]$ ):

$$
u(x, y)=\frac{y^{n}}{(n-1) !} \sum_{k=0}^{n-1}(-1)^{k} C_{(n-1)}^{k} \frac{\partial^{k}}{\partial y^{k}}\left[\frac{P f_{n-1-k}}{y}\right],
$$

where

$$
\operatorname{Pf}(x)=\frac{y}{\pi} \int_{\mathbb{R}} \frac{f(t) d t}{(t-x)^{2}+y^{2}}
$$

and for $u(x, y)$ to vanish at infinity the given functions should satisfy the following integral conditions:

$$
\int_{\mathbb{R}} t^{l} f_{k}(x) d x=0, \quad l=0,1, \ldots, 2(m-1), \quad k=1,2, \ldots n-1 .
$$

where $k=2 m$, or $k=2 m-1$. The above problems for any $l$ can be solved by the same technique. That is why it is sufficient to consider the case $l=0$. Thus, all $f_{k}(x)$ $\left(k=1,2, \ldots n-1, \quad x \in \mathbb{R}\right.$ are known and $u(x, 0)=f_{0}(x)$ is the unknown function. If the latter function is defined, the solution of the problem 1 will be represented in the form (25). By virtue of (22) and (25), for unknown function $f_{0}(x) \equiv \varphi(x), x \in \mathbb{R}$ one can easily obtain dual integral equations of the form (3), where

$$
k_{j}(x)=\left.\frac{h_{j}^{n}(-1)^{n-1}}{(n-1) ! \pi} \frac{\partial^{n-1}}{\partial y^{n-1}}\left[\frac{1}{x^{2}+y^{2}}\right]\right|_{y=h_{j}}, \quad j=1,2 ; x \in \mathbb{R} .
$$

It is not difficult to obtain that

$$
F_{t}\left[k_{j}(x)\right]=\left.\frac{h_{j}^{n}(-1)^{n-1}}{(n-1) ! \sqrt{2 \pi}} \frac{\partial^{n-1}}{\partial y^{n-1}}\left[\frac{e^{-|t| y}}{y}\right]\right|_{y=h_{j}}, \quad j=1,2 ; t \in \mathbb{R} .
$$

As the latter is an even function with respect to $t$, the conditions (17) and (18) will have the simplest form:

$$
F_{1}^{+}(t)+F_{2}^{+}(t)=F_{1}^{-}(t)+F_{2}^{-}(t)+g(t), \quad t \in \mathbb{R},
$$


or

$$
F_{1}^{+}(t)-F_{2}^{+}(t)=-\left(F_{1}^{-}(t)-F_{2}^{-}(t)\right)+g(t), \quad t \in \mathbb{R},
$$

The piecewise functions $F_{1}(z)+F_{2}(z)$ or $F_{1}(z)-F_{2}(z)$ by these conditions, as is well known [4], are defined uniquely and written immediatly using Cauchy type integrals. After this $\varphi(x)$ will be defined uniquely too.

Solution of the problem 2. The solution of the classical problem for (20) in $D$ when on $\mathbb{R}$ is given $(2.4)$ for all $k=0,1, \ldots, n-1$ can be written in the form (see, for example, $\left.\left[5_{a}\right]\right)$

$$
u(x, y)=P f_{0}+\frac{y}{\pi} \sum_{k=1}^{n-1} \frac{1}{4^{k}[(k-1) !]^{2} k} \int_{\mathbb{R}} f_{k}(t) r^{2(k-1)} \ln r^{2} d t
$$

where $r^{2}=(x-t)^{2}+y^{2}$, and for $u(x, y)$ to vanish at infinity the given functions should satisfy the conditions:

$$
\int_{\mathbb{R}} t^{l} f_{k}(t) d t=0, k=1,2, \ldots, n-1, \quad l=0,1, \ldots, 2(k-1) .
$$

As in (23) we consider $l=0$, it means that $f_{0}(x) \equiv \varphi(x)$ is unknown and by virtue of (23), (32), it will be the solution of dual integral equations (3), where the kernels are defined by $(28)$ with $k=1$. It means that in this case the piecewise functions $F_{1}(z)+F_{2}(z)$ or $F_{1}(z)-F_{2}(z)$ will be defined again by the conditions $(30)(31)$. Then it is understood how to define $f_{0}(x)$.

Solution of problem 3. To solve this problem we need to define $u(x, 0)$ for $x>0$. Taking into consideration $(2.5)$, (25) for unknown function $u(x, 0) \equiv \varphi(x), x>0$ one can receive the integral equation of the form (1), where $k(x)$ is defined by (28) with $h_{j}=h$. The solution of (1) when $m^{2}=1$ is represented explicitly in [2], [6].

Note that for (20) in $D$ one can consider various classical boundary conditions, for example, when on $\mathbb{R}$ are given the quantities

$$
\left.\frac{\partial^{l} u}{\partial y^{l}}\right|_{y=0}=f_{l}(x), \quad l=0,1, \ldots, k,\left.\quad \Delta^{p} u\right|_{y=0}=f_{p}(x), \quad p=k+1, \ldots, n-1
$$

and corresponding nonlocal problems can be posed and solved quite analogous way as above. For instance, biharmonic in $D$ the function $u(x, y)$ vanishing at infinity with the conditions

$$
\left.\frac{\partial u}{\partial y}\right|_{y=0}=f_{0}(x),\left.\quad \Delta u\right|_{y=0}=f_{1}(x), \quad x \in \mathbb{R},
$$

will be defined as

$$
u(x, y)=\frac{1}{2 \pi} \int_{\mathbb{R}}\left[f_{0}(t) \ln \left[(x-t)^{2}+y^{2}\right]-(x-t) f_{1}(t) \operatorname{arctg} \frac{y}{x-t}\right] d t,
$$

where $f_{0}, f_{1}$ by force of the condition at infinity must satisfy the equalities:

$$
\int_{\mathbb{R}} f_{0}(t) d t=0, \quad \int_{\mathbb{R}} f_{1}(t) t^{l} d t=0, \quad l=1,2 .
$$

Then, with the conditions

$$
\left.u\right|_{y=0}=f_{0}(x),\left.\quad \frac{\partial \Delta u}{\partial y}\right|_{y=0}=f_{1}(x), \quad x \in \mathbb{R}
$$


will be defined as

$$
u(x, y)=p f_{0}+\frac{y}{4 \pi} \int_{\mathbb{R}} f_{1}(t)\left[y \ln \left[(x-t)^{2}+y^{2}\right]+2(x-t) \operatorname{arctg} \frac{y}{x-t}\right] d t
$$

where $f_{1}$ must satisfy conditions $(36)$ for $l=0,1,2$.

Now, if in (34) or in (37) the first condition is replaced by the nonlocal conditions of the form (22), then corresponding representations (35) or (38) will give us for the unknown function $f_{0}(x), \quad x \in \mathbb{R}$ dual integral equations of the form (3). As $f_{0}(x)$ will be defined with the help of $f_{1}(x)$, it is obvious, the condition (36) will be accomplished if $f_{1}(x)$ must satisfy the corresponding supplementary condition. Thus, many interesting problems can be solved by above considered way.

\section{References}

[1] V. I. Azamatova, Integral equations with the difference and summary kerns in the space $(\alpha, \beta)$, Izv. AN BSSR Phys. Math. No. 5 (1972), 24-33 (Russian).

[2] F. D. Berkovic, An integral equation on the semi-axis, Izv. Vyssh. Uchebn. Zaved. Mathem. 1966 no. 1 (50), 3-14. (Russian).

[3] F. D. Gakhov, U. I. Cherski, Equations of convolution type, (Russian), "Nauka", Moscow, 1978.

[4] N. I. Muskhelishvili, Singular integral equations, Groningen, Noordhoff, 1963.

[5a] E. I. Obolashvili, Nonlocal problems for some partial differential equations, Applicable Analysis, vol. 45, 1992, 269-280.

[5b] - Nonlocal problems for the Beltrami Equation and Polyharmonic Functions, Proceedings devoted to N. I. Muskhelishvili's anniversary, Tbilisi 1994.

[6] V. V. Sobolev, Diffuse irradiation in a medium with mirror reflection boundaries, DAN SSSR 136 (1961) 571-574 (Russian).

[7] E. C. Titchmarsh, Introduction to the Theory of Fourier Integrals, Oxford 1950. 